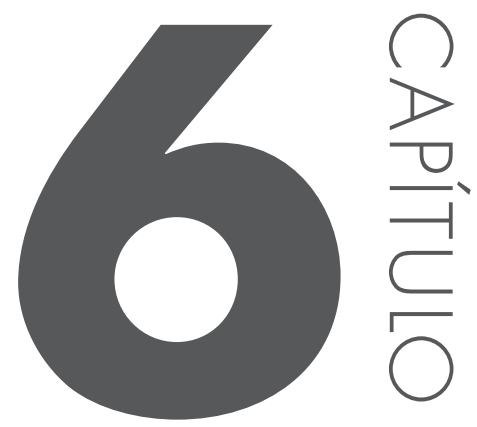

\title{
RESULTADOS OBTIDOS
}

Nesta seção são apresentados os resultados obtidos com cada EPG, comparando os resultados calculados com os resultados obtidos experimentalmente por NINE (1978) e por distintas simulações computacionais.

Com a intenção de facilitar a solução da EPG obtida, um aplicativo para dispositivos móveis foi criado, sendo uma importante ferramenta facilitadora para se estimar o valor da FR dos freios, tendo em vista que se trata de um aplicativo interativo e compatível com a maior parte dos tablets e smarthphones comercializados. 


\subsection{VALIDAC̣ÃO DAS EQUAC̣ÕES COM OS DADOS EXPERIMENT́AIS}

Como o primeiro passo para a validação das equações obtidas, foi realizada a comparação das respostas destas, com os dados experimentais de Nine (1978). Conforme demonstra a Tabela 14.

Tabela 14 - Validação das EPGs obtidas, com os dados experimentais

\begin{tabular}{|c|c|c|c|c|c|c|}
\hline \multirow{2}{*}{ Teste } & \multicolumn{2}{|c|}{ (NINE 1978) } & \multicolumn{2}{|c|}{$\mathrm{FP}=50 \mathrm{kN}$} & \multicolumn{2}{|c|}{$\mathrm{FP}=100 \mathrm{kN}$} \\
\hline & FP & FR & FR (EPG) & Desvio \% & FR (EPG) & Desvio \% \\
\hline 1 & 72 & 94 & 88 & $-7 \%$ & 81 & $-14 \%$ \\
\hline 2 & 58 & 60 & 57 & $-5 \%$ & 47 & $-21 \%$ \\
\hline 3 & 66 & 66 & 67 & $2 \%$ & 57 & $-14 \%$ \\
\hline 4 & 56 & 64 & 58 & $-9 \%$ & 47 & $-27 \%$ \\
\hline 5 & 96 & 114 & 117 & $3 \%$ & 112 & $-2 \%$ \\
\hline 6 & 70 & 80 & 74 & $-7 \%$ & 65 & $-18 \%$ \\
\hline 7 & 68 & 80 & 73 & $-8 \%$ & 65 & $-19 \%$ \\
\hline 8 & 60 & 66 & 65 & $-2 \%$ & 48 & $-27 \%$ \\
\hline 9 & 84 & 114 & 120 & $5 \%$ & 109 & $-5 \%$ \\
\hline 10 & 68 & 78 & 82 & $5 \%$ & 64 & $-18 \%$ \\
\hline 11 & 70 & 78 & 81 & $4 \%$ & 60 & $-24 \%$ \\
\hline 12 & 84 & 98 & 107 & $9 \%$ & 91 & $.7 \%$ \\
\hline 13 & 116 & 168 & 184 & $10 \%$ & 173 & $3 \%$ \\
\hline 14 & 68 & 82 & 81 & $.1 \%$ & 62 & $-23 \%$ \\
\hline 15 & 64 & 74 & 71 & $-4 \%$ & 58 & $-21 \%$ \\
\hline 16 & 96 & 128 & 135 & $5 \%$ & 123 & $-4 \%$ \\
\hline 17 & 80 & 100 & 98 & $-2 \%$ & 99 & $-1 \%$ \\
\hline 18 & 116 & 160 & 179 & $11 \%$ & 158 & $-2 \%$ \\
\hline 19 & 98 & 114 & 128 & $11 \%$ & 124 & $9 \%$ \\
\hline Desvio médio & - & - & - & $5 \%$ & - & $13 \%$ \\
\hline
\end{tabular}

Comparando-se os resultados obtidos por cada uma das equações, observase que a EPG desenvolvida com $\mathrm{FP}=50 \mathrm{KN}$, apresentou maior proximidade dos valores obtidos experimentalmente, que a EPG elaborada com $\mathrm{FP}=100 \mathrm{kN}$.

A média dos valores absolutos das diferenças percentuais, para a equação elaborada com FP igual a $50 \mathrm{KN}$, foi igual a $5 \%$, resultado muito satisfatório, quando comparado com a média obtida pela equação elaborada com FP igual a $100 \mathrm{kN}$, que foi de $13 \%$.

Com o intuito, de se validar a equação elaborada com FP igual a $50 \mathrm{kN}$, observou-se o seu comportamento com outros testes. Foram feitas simulações de vinte casos escolhidos, para se testar as respostas fornecidas pela EPG, consi- 
derando-se os respectivos limites de validade estabelecidos para cada $\pi$-termo. A Tabela 15 apresenta os valores atribuídos para todos os parâmetros e a Tabela 16, os resultados comparativos da EPG com os dados obtidos pelas simulações no STAMPACK ${ }^{\circledR}$.

Tabela 15 - Parâmetros utilizados para o teste da EPG

\begin{tabular}{|c|c|c|c|c|c|c|c|c|c|c|c|}
\hline Teste & $\underset{(\mathrm{mm})}{t}$ & $\begin{array}{l}\text { Rd } \\
\text { (mm) }\end{array}$ & $\underset{(\mathrm{mm})}{\mathrm{C}}$ & n & $\begin{array}{c}E \\
(\mathrm{MPa})\end{array}$ & $\begin{array}{c}K \\
(\mathrm{MPa})\end{array}$ & $\begin{array}{c}\mathrm{SY}^{\mathrm{y}} \\
(\mathrm{MPa})\end{array}$ & $\mu$ & $\begin{array}{l}\mathrm{Rm} \\
(\mathrm{mm})\end{array}$ & $\begin{array}{c}h \\
(\mathrm{~mm})\end{array}$ & $\begin{array}{l}\text { FP } \\
\text { (kN) }\end{array}$ \\
\hline 1 & 0,76 & 6,50 & 1,04 & 0,23 & 206.000 & 516 & 171,7 & 0,170 & 6,50 & 10,54 & 50 \\
\hline 2 & 0,76 & 4,75 & 0,76 & 0,23 & 206.000 & 520 & 171,7 & 0,170 & 4,75 & 7,70 & 63 \\
\hline 3 & 0,81 & 4,75 & 0,76 & 0,26 & 71.000 & 643 & 192,9 & 0,300 & 5,50 & 11,81 & 75 \\
\hline 4 & 0,80 & 5,00 & 0,85 & 0,44 & 110.000 & 530 & 69,0 & 0,050 & 6,00 & 8,00 & 65 \\
\hline 5 & 0,76 & 4,74 & 0,76 & 0,23 & 206.000 & 510 & 170,0 & 0,300 & 4,75 & 7,70 & 75 \\
\hline 6 & 0,77 & 4,75 & 0,77 & 0,22 & 210.000 & 516 & 171,7 & 0,180 & 4,75 & 5,00 & 50 \\
\hline 7 & 0,78 & 4,75 & 0,78 & 0,17 & 70.000 & 680 & 40,0 & 0,030 & 4,75 & 7,20 & 40 \\
\hline 8 & 0,82 & 5,50 & 0,84 & 0,21 & 97.000 & 585 & 75,0 & 0,100 & 5,00 & 9,00 & 62 \\
\hline 9 & 0,76 & 4,75 & 1,25 & 0,23 & 206.000 & 519 & 171,7 & 0,150 & 4,75 & 7,72 & 50 \\
\hline 10 & 0,74 & 5,00 & 0,82 & 0,30 & 207.000 & 545 & 138,0 & 0,120 & 5,00 & 7,00 & 60 \\
\hline 11 & 0,79 & 4,90 & 0,79 & 0,18 & 72.000 & 600 & 45,0 & 0,110 & 6,00 & 8,70 & 60 \\
\hline 12 & 0,79 & 4,90 & 0,79 & 0,20 & 170.000 & 510 & 110,0 & 0,110 & 6,00 & 8,70 & 75 \\
\hline 13 & 0,76 & 4,75 & 0,76 & 0,23 & 206.000 & 516 & 255,0 & 0,170 & 4,75 & 7,70 & 100 \\
\hline 14 & 0,85 & 5,75 & 0,88 & 0,20 & 71.000 & 640 & 50,0 & 0,180 & 5,50 & 11,80 & 68 \\
\hline 15 & 0,85 & 5,75 & 0,88 & 0,25 & 192.000 & 490 & 150,0 & 0,090 & 5,50 & 11,80 & 72 \\
\hline 16 & 0,76 & 4,75 & 1,20 & 0,23 & 206.000 & 520 & 177,0 & 0,180 & 4,75 & 7,70 & 97 \\
\hline 17 & 0,90 & 6,70 & 0,92 & 0,17 & 69.000 & 525 & 95,0 & 0,070 & 5,50 & 10,00 & 54 \\
\hline 18 & 0,90 & 6,70 & 0,92 & 0,25 & 210.000 & 510 & 180,0 & 0,150 & 5,50 & 10,00 & 90 \\
\hline 19 & 0,76 & 4,70 & 0,76 & 0,23 & 206.000 & 516 & 250,0 & 0,170 & 4,70 & 7,71 & 88 \\
\hline 20 & 0,97 & 5,50 & 0,97 & 0,24 & 207.000 & 531 & 173,0 & 0,070 & 5,50 & 11,97 & 98 \\
\hline
\end{tabular}

O comparativo dos resultados simulados e calculados pela EPG pode ser observado na Tabela 16 abaixo. 
Tabela 16 - Comparativo dos resultados obtidos or meio da simulação em EF e calculados pela EPG

\begin{tabular}{|c|c|c|c|c|}
\hline \multirow{2}{*}{ Teste } & \multicolumn{2}{|c|}{ Stampack } & \multicolumn{2}{|c|}{ EPG (FP=50kN) } \\
\hline & FP & FR & EPG & Desvio \% \\
\hline 1 & 50 & 64 & 62 & $3 \%$ \\
\hline 2 & 63 & 86 & 91 & $6 \%$ \\
\hline 3 & 75 & 120 & 110 & $9 \%$ \\
\hline 4 & 65 & 54 & 55 & $3 \%$ \\
\hline 5 & 75 & 124 & 129 & $4 \%$ \\
\hline 6 & 50 & 62 & 61 & $1 \%$ \\
\hline 7 & 40 & 17 & 18 & $4 \%$ \\
\hline 8 & 62 & 55 & 52 & $5 \%$ \\
\hline 9 & 50 & 68 & 66 & $2 \%$ \\
\hline 10 & 60 & 73 & 75 & $3 \%$ \\
\hline 11 & 60 & 31 & 34 & $10 \%$ \\
\hline 12 & 75 & 88 & 90 & $3 \%$ \\
\hline 13 & 100 & 126 & 140 & $11 \%$ \\
\hline 14 & 68 & 45 & 43 & $5 \%$ \\
\hline 15 & 72 & 87 & 90 & $3 \%$ \\
\hline 16 & 97 & 122 & 136 & $10 \%$ \\
\hline 17 & 54 & 43 & 47 & $7 \%$ \\
\hline 18 & 90 & 110 & 119 & $7 \%$ \\
\hline 19 & 88 & 116 & 124 & $7 \%$ \\
\hline 20 & 98 & 118 & 128 & $8 \%$ \\
\hline Desvio médio & - & - & - & $5 \%$ \\
\hline
\end{tabular}

Nota-se que a EPG escolhida, respondeu com precisão aos testes efetuados. O desvio máximo obtido foi de $11 \%$ e a média dos valores absolutos das diferenças percentuais foi de $5 \%$, com desvio padrão de $3 \%$. Estes resultados indicam, que as curvas obtidas para os $\pi$-termos foram bem estabelecidas para esta equação e a metodologia bem empregada, tendo em vista que este valor é inferior aos anteriores divulgados na literatura sobre o assunto e é inferior aos limites fornecidos pela medição gráfica fornecida pelo código explícito utilizado.

\subsection{DESENVOLVIMENTO DO APLICATIVO PARA CÁLCULO}

Após realizar os testes e validar as EPG, pensou-se em uma forma de facilitar esta rotina relativamente extensa de cálculo, necessária para se obter matematicamente a resposta de FR para os freios. Para isso, foi criado um aplicativo para dispositivos móveis de comunicação, tais como: smatphones e tablets. Este apli- 
cativo é compatível com a plataforma Android e foi programado utilizando-se a linguagem Adobe Actionscript 3.0.

\subsubsection{ESCOLHA DA PLATAFORMA E LINGUAGEM DE PROGRAMAÇÃO}

A fim de se facilitar o processo de obtenção da FR por meio da solução da EPG determinada, foi projetado um aplicativo de cálculo compatível com a plataforma Android.

Esta plataforma foi definida, por meio da sua presença no mercado global e compatibilidade com a maior parte de smartphones e um grande volume de tablets comercializados no Brasil e no mundo.

Segundo o relatório Worldwide Smartphone 2013-2017 Forecast and Anal$y$ sis do IDC (International Data Corporation), consultoria americana especializada em análise de mercado e tecnologia, o sistema Android representa hoje 52\% das vendas de smartphones no mundo, IOS (plataforma dos aparelhos Apple) $38 \%$ e os demais $10 \%$. Para os tablets a concorrência fica mais próxima e com uma pequena vantagem para os IOS, que representam 53\% enquanto o Android representam $43 \%$ e os demais $4 \%$.

O programa foi feito no recurso AIR (Adobe Integrated Runtime) para Android é um programa multiplataforma de ambiente tempo de execução desenvolvida pela Adobe Systems para construir aplicações de Internet usando Adobe Flash, Adobe Flex, HTML, ou AJAX. A programação foi feita utilizando-se o Adobe Actionscript 3.0 (AS3.0), que é a linguagem de programação orientada a objetos, da plataforma Adobe Flash. Esta é uma linguagem de script baseada no ECMAScript (linguagem usada para web, possui referência para javaScript), basicamente associada como um script de comportamento da aplicação Adobe Flash.

Esta linguagem, ajuda a prover uma excelente experiência de interatividade, nos projetos construídos no Adobe Flash, além de um suporte de conexão pela web, graças a um completo conjunto de componentes.

\subsubsection{APRESENTAÇÃO DO APLICATIVO}

Como dito anteriormente, o aplicativo foi programado no recurso AIR do programa Adobe Flash CS5.5, utilizando a linguagem AS3.0.

Os campos de entrada de dados foram definidos e é por meio, de cada um deles, que o usuário pode alterar um parâmetro e verificar a resposta calculada para a FR. Os cálculos podem ser corretamente realizados quando o usuário fornece todos os parâmetros requeridos. O programa é responsável por verificar 
cada um destes parâmetros e calcular a resposta com base na EPG programada. Neste caso, foi à equação que obteve melhores resultados na validação, ou seja, a construída com as simulações que utilizaram FP igual a $50 \mathrm{kN}$.

Ao aplicativo foi dado o nome de "Drawbead Calc", e sua interface pode ser vista na Figura 54.

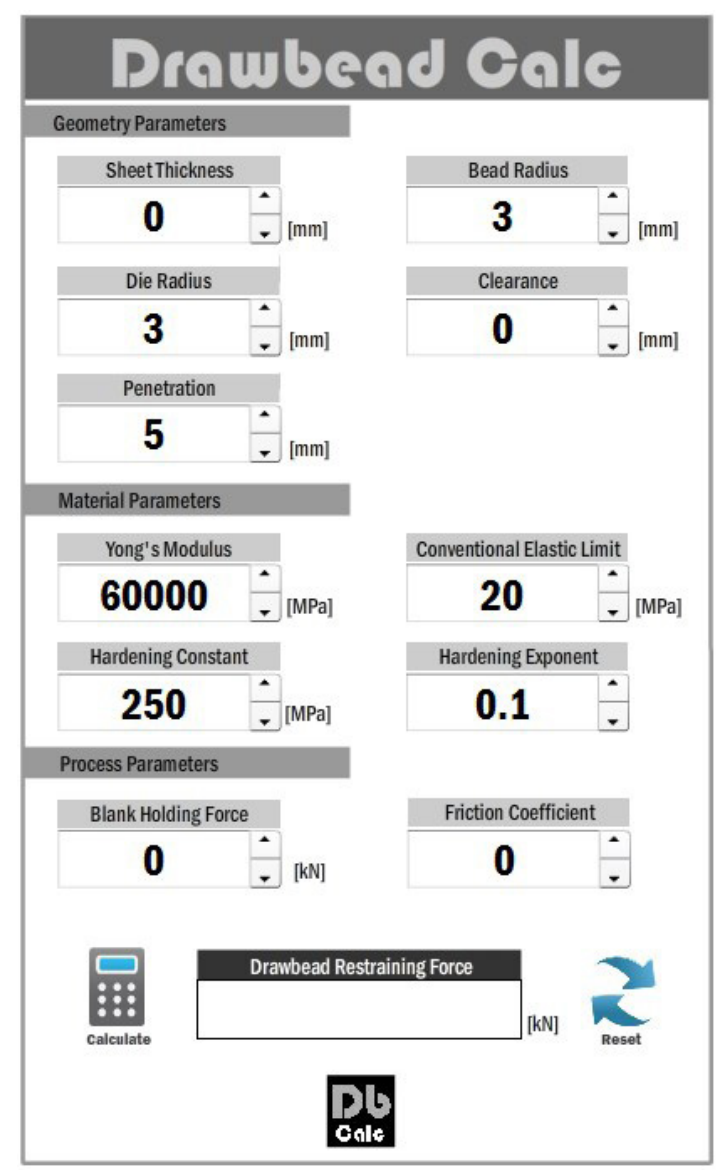

Figura 54 - Interface do Aplicativo "Drawbead Calc"

A intenção da criação desta ferramenta é a de auxiliar os usuários com os cálculos da FR, onde, de forma simples e dinâmica, podem-se alterar os parâmetros e verificar a reposta obtida, com o simples auxilio de um dispositivo com plataforma Android. Além disso, busca demonstrar a sinergia obtida entre empresa e o meio acadêmico, para o desenvolvimento de novas tecnologias, por meio de parcerias que buscam desenvolver um bem comum. 